\title{
Günter Stemberger
}

\section{Die Umformung des palästinischen Judentums nach 70: Der Aufstieg der Rabbinen}

Eine für das Verständnis der jüdischen Geschichte zentrale Frage ist, wie aus dem palästinischen Judentum der Zeit vor 70, einer von Tempel und Priestertum dominierten Gesellschaft, ein Gemeinwesen geworden ist, in dem die Rabbinen das jüdische Leben bestimmten. Geschah dies unvermittelt und plötzlich oder war dies nur das Ergebnis einer langen, vielleicht Jahrhunderte währenden Entwicklung? Sind die Rabbinen als die direkten Erben der Pharisäer zu betrachten? Wenn ja, welches Erbe konnten sie antreten? Wieviel Einfluß hatten die Pharisäer vor 70 ? Wurden die Führer der rabbinischen Schulen unmittelbar oder zumindest sehr bald nach 70 als die Führer des Judentums vom jüdischen Volk und von der römischen Verwaltung anerkannt? Wie schnell gab die alte Führung ihre Machtansprüche auf? Diese und ähnliche Fragen scheinen erst in den letzten Jahrzehnten drängend geworden zu sein; denn wenn man Schürers Geschichte in ihrer letzten deutschen Fassung mit der englischen Neubearbeitung siebzig Jahre später vergleicht, findet man auf den einschlägigen Seiten keine wesentlichen Unterschiede. Die Einstellung des alten wie des neuen Schürer mögen einige charakteristische Zitate zum Umbruch im Jahr 70 kennzeichnen:

„In das Erbe der Sadducäer und Priester traten die Pharisäer und Rabbinen. Sie waren vortrefflich auf den Antritt dieser Erbschaft vorbereitet. Schon seit zwei Jahrhunderten waren sie mehr und mehr zur führenden Macht geworden. Nun fiel ihnen mit einem Male die Alleinherrschaft zu. [Der Untergang Jerusalems bedeutet nicht mehr und nicht weniger als die Auslieferung des Volkes an den Pharisäismus und die Rabbinen]; denn die Factoren, welche diesen bisher noch entgegengestanden hatten, waren zur Bedeutungslosigkeit herabgesunken."

1 Emil Schürer, Geschichte des jüdischen Volkes im Zeitalter Jesu Christi, 3 Bde. (Leipzig 31901-1909) I 656 = Emil Schuirer, The History of the Jewish People in the Age of Jesus Christ (175 B.C.-A.D. 135). A New English Version Revised and edited by Geza Vermes, Fergus Millar, Matthew Black (ab Bd. 3 Martin Goodman) 3 Bde. (Edinburgh 1973-1987) I 524. Der einzige Unterschied zwischen den beiden Fassungen ist, daß im Englischen der im Zitat in eckige Klammern gesetzte Satz ausgelassen wurde - man hat ihn offenbar als anstößig empfunden. Im folgenden wird das Werk einfach als Schürer mit dem Zusatz G[eschichte] bzw. $H$ [istory] angeführt. 
„Diese Gelehrten ... bildeten nun noch ausschliesslicher und unumschränkter als ehedem die höchsten Autoritäten des Volkes ... Aller Eifer der Frommen musste sich darauf beschränken, zu thun, was die Rabbinen ihnen vorschrieben. Es bedurfte keines äusseren Zwanges ... Ja man erkannte sie nicht nur als Gesetzgeber in geistlichen und weltlichen Dingen an, sondern man unterwarf sich auch ihrem Richterspruch in streitigen Fällen, selbst in Fragen des Mein und Dein... Das höchste Ansehen genoss gegen Ende des ersten und im Anfang des zweiten Jahrhunderts nach Chr. der Gerichtshof ${ }^{2}$ zu Jamnia (Jabne), ein Kollegium von Gelehrten, das schwerlich von der römischen Behörde eine weitgehende Autorisation hatte ${ }^{3}$, das aber thatsächlich an die Stelle des alten Synedriums von Jerusalem trat als oberster Gerichtshof von Israel ... Überhaupt galten die in Jabne getroffenen gesetzlichen Entscheidungen als normativ. Ja die Succession Jabne's in die Rechte von Jerusalem wurde so sehr als die Regel angesehen, dass man es als eine Ausnahme constatirte, wo dies nicht der Fall war." 4

Die englische Fassung läßt dann einen Satz des deutschen Textes aus: „Man darf annehmen, dass dieser ,Gerichtshof' von Jabne die vom jüdischen Volk ihm freiwillig zugestandene Autorität nicht nur auf dem Gebiete des Ceremonialgesetzes, sondern auch auf dem Gebiete des Civil- ja des Criminalrechtes zur Anwendung gebracht hat." Diese Auslassung geht aber offenbar nicht auf eine Meinungsverschiedenheit zwischen Schürer und seinen Bearbeitern zurück; vielmehr wurde der Text als redundant betrachtet, denn der englische Text fährt in Übereinstimmung mit dem deutschen fort: „Im Civilrecht mag er dazu, nach Lage der allgemeinen Gesetzgebung, wirklich befugt gewesen sein... Aber in Criminalsachen wird diese Gerichtsbarkeit mehr eine usurpirte als eine vom Kaiser zugestandene gewesen sein. "5

Man könnte es als kleinen Widerspruch innerhalb des Textes betrachten, wenn es zuerst heißt, das Gelehrtenkollegium von Jabne habe „schwerlich von der römischen Behörde eine weitgehende Autorisation" gehabt, dann aber, daß es zum Gericht ,im Civilrecht... nach Lage der allgemeinen Gesetzgebung, wirklich befugt gewesen sein “ könnte ${ }^{6}$. Im allgemeinen ist die Position aber klar: Die Rabbinen übernahmen sofort nach 70 die ganze Macht, vom jüdischen Volk voll angenommen und, zumindest in Fragen des Zivilrechts, auch von der römischen Verwaltung bevollmächtigt. Ich glaube nicht, daß heute, 25 Jahre nach der Überarbeitung des ersten Bandes, viele Gelehrte diese Positionen bejahen würden. Auf den

2 Englisch: „academy“.

${ }^{3}$ Englisch: "with scarcely any proper authorization from the Roman administration".

4 Schürer, G I $657 \mathrm{f}$. = H I $525 \mathrm{f}$.

5 Schürer, G I $658=$ H I 526.

6 Schürer, G I 658, Anm. 39 = H I 526, Anm. 64 kommentiert die in mEd 7,7 erwähnte Reise Gamaliels II. nach Syrien, „um eine Erlaubnis ... zu erlangen“ (לטול רטות): „Es ist möglich, dass es sich dabei um Verleihung oder Erweiterung oder Ausübung richterlicher Befugnisse handelte." 
folgenden Seiten möchte ich nur kurz auf die wesentlichen Unterschiede zwischen den in Schürer vertretenen Meinungen und neuerer Forschung und auf die Gründe dafür hinweisen.

\section{Pharisäer und Rabbinen: Wieviel Kontinuität?}

Für Schürer und seine Bearbeiter besteht kein Zweifel, daß zwischen beiden Gruppen eine klare Verbindung besteht. Ja, man betrachtet sie als miteinander identisch; nur die Namen haben gewechselt - die pharisäischen Toragelehrten heißen jetzt Rabbinen. Das wird aus dem folgenden Zitat über die Lage nach 70 ganz deutlich: „Die pharisäischen Gesetzeslehrer, die schon im letzten Jahrhundert vor der Zerstörung des Tempels tatsächlich den größten Einfluß ausgeübt hatten, wurden die alleinigen Führer des Volkes. Der politische Untergang hatte also direkt zur Folge eine Steigerung der rabbinischen Macht und einen Aufschwung der schriftgelehrten Studien. "7

Im gründlicher überarbeiteten Kapitel über die Pharisäer begegnen wir derselben Position in einem Abschnitt, der kein Gegenstück im deutschen Text hat: „after the destruction of the Temple, the survivors of the Pharisaic movement became the only dominant force in the life of the Palestinian Jewish community, and were solely responsible for the revival and recodification of the ancestral traditions in the form of rabbinic Judaism. "8 Das entspricht völlig einem verbreiteten Konsens, wonach man gewohnt ist, vom „pharisäisch-rabbinischen Judentum “ $\mathrm{zu}$ sprechen.

Unter modernen Autoren hat diese Auffassung eine zusätzliche Stütze durch die einflußreiche Studie von Morton Smith, „Palestinian Judaism in the First Century", erhalten. Smith verstand, wie ja bekannt ist, die Tatsache, daß Josephus den Pharisäern in seinen Antiquitates so viel mehr Gewicht als im früheren Bellum einräumt und hier so sehr ihren Einfluß auf die Volksmassen betont, als Plädoyer gegenüber den römischen Behörden: „The Pharisees, he says, again and again, have by far the greatest influence with the people. Any government which secures their support is accepted; any government which alienates them has trouble."

Die These von Smith ist durch Jacob Neusner populär geworden ${ }^{10}$. Dieser Auffassung gegenüber zu Recht kritisch sind dagegen unter anderen David Good-

7 Schürer, G II $432=\mathrm{H}$ II 369.

8 Schürer, H II 402 f.; cf. G II 475.

${ }^{9}$ Morton Smith, Palestinian Judaism in the First Century, in: Moshe Davis (Hrsg.), Israel: Its Role in Civilization (New York 1956) 67-81, 76; Nachdruck: Morton Smith, Studies in the Cult of Yahweh, Bd. 1 (Religions in the Graeco-Roman World 130, Leiden 1996) 104-115, 111.

10 Jacob Neusner, Josephus's Pharisees, in: Louis Feldman, Gobei Hata (Hrsg.), Josephus, Judaism and Christianity (Leiden 1987) 274-292; fast dasselbe schon in: Ex Orbe Religionum: Studia Geo Widengren Oblata (Leiden 1972) I 224-244. 
blatt ${ }^{11}$ und Steve Mason ${ }^{12}$. Es stimmt zwar, daß Josephus nur in den Antiquitates den Einfluß der Pharisäer über die Massen hervorhebt. Doch ist das Bild, das er zeichnet, durchaus nicht reine Propaganda für die Pharisäer. Die Pharisäer, die Josephus in den Antiquitates schildert, haben zwar großen Einfluß, mißbrauchen ihn aber ständig, um Schwierigkeiten zu machen, und das auch unter Herrschern wie Salome Alexandra, die ihnen alle Macht überlassen hatte, oder unter Herodes, der ihnen so freundlich gesinnt war. Josephus verweist auch auf ihre geistige Verwandtschaft mit den Zeloten und nennt in seiner Vita sogar einige Pharisäer beim Namen, die an vorderster Front am Aufstand beteiligt waren. Die Gemäßigten unter ihnen hatten dagegen nicht genügend Einfluß auf das Volk, um es im entscheidenden Jahr 66 vom Aufstand abzuhalten. Alles in allem empfiehlt das in den Antiquitates gebotene Pharisäerbild diese nicht unbedingt als die neue Führung, auf die sich die Römer verlassen könnten.

Die Darstellung der Pharisäer im späteren Werk des Josephus ist also nicht als Propaganda für sie zu verstehen. Könnte man aber nicht doch die Tatsache, daß ihnen Josephus hier im Vergleich zum früheren Bellum um so viel mehr Aufmerksamkeit widmet, zumindest als Hinweis darauf sehen, daß die Pharisäer zwanzig Jahre nach dem Krieg die beherrschende Kraft im palästinischen Judentum geworden sind? Das von Josephus in seinem Spätwerk gebotene Bild scheint ja in den Evangelien eine Bestätigung zu erhalten. Auch hier nimmt die Bedeutung der Pharisäer in den späteren Texten zu. In Markus, dem ältesten Evangelium, begegnen uns die Pharisäer mehrmals als Gegner Jesu in Galiläa; während Jesu Aufenthalt und Passion in Jerusalem werden sie dagegen nie genannt. Dies ist auffällig; denn in Wirklichkeit war Jerusalem das Zentrum der Pharisäer, während sie in Galiläa kaum in Erscheinung traten. In Matthäus sind die Pharisäer die stets gegenwärtigen Gegner Jesu; Mt 23 ist ein scharfer Angriff auf die „Schriftgelehrten und Pharisäer" - doch ihre Lehrautorität wird in 23,2f anerkannt. Lukas bietet ein differenziertes Bild und schildert mehrere Pharisäer, die Jesus nahestehen; in der Apostelgeschichte verweist er auf die Gemeinsamkeiten zwischen Pharisäern und Christen. Im jüngsten Evangelium, Johannes, sind die Pharisäer die eigentlichen Vertreter des jüdischen Volkes, die treibende Kraft in diesem, die wesentlichen, wenn nicht die einzigen Feinde der christlichen Bewegung ${ }^{13}$.

Die meisten Gelehrten sind der Meinung, daß die Evangelien nicht die geschichtlichen Verhältnisse zur Zeit der öffentlichen Tätigkeit Jesu spiegeln, sondern die Umstände, unter denen die frühen christlichen Gemeinden lebten und die Evangelientraditionen Gestalt annahmen. Besonders hinsichtlich des vierten Evangeliums sind viele Autoren geneigt anzunehmen, daß Johannes die Situation seiner eigenen Gemeinde Ende des ersten Jahrhunderts in das Leben Jesu rück-

11 David Goodblatt, The Place of the Pharisees in First Century Judaism: The State of the Debate, in: JSJ 20 (1989) 12-30.

12 Steve Mason, Flavius Josephus on the Pharisees. A Comparison-Critical Study (SPB 39, Leiden 1991) 32-36.

${ }^{13}$ Für eine kurze Zusammenfassung siehe Günter Stemberger, Pharisäer, Sadduzäer, Essener (SBS 144, Stuttgart 1991) 24-39. 
projizierte ${ }^{14}$. Sein Evangelium würde demnach historische Verhältnisse schildern, in denen die Pharisäer (oder ihre Erben in den Jahrzehnten nach 70) die einzigen jüdischen Gegner der johanneischen Gemeinde waren. Das Johannesevangelium wird dann gerne als Reaktion auf rabbinische Entscheidungen in Jabne verstanden (die sogenannte „Synode von Jamnia“15).

Die verbreitete Meinung, daß die Rabbinen direkt die pharisäische Bewegung fortführen, hat Clemens Thoma griffig formuliert: „Der Rabbinismus ist der aus einer Gruppenexistenz in die Gesamtverantwortung für das Judentum hineingezogene Pharisäismus." 16 Zur größeren Klarheit sollte man zwei Aspekte dieser Position unterscheiden - die persönliche und ideologische Kontinuität von Pharisäern und Rabbinen auf der einen Seite, und den tatsächlichen Einfluß der frühen rabbinischen Bewegung auf der anderen. Der zweite Aspekt ist zusammen mit den allgemeineren Fragen der Organisation und Struktur des Judentums nach 70 zu sehen, mit der Frage nach dem Aufkommen des Patriarchats und anderer Führungseinrichtungen; dies soll im folgenden Abschnitt besprochen werden. Hier geht es uns nur um den ersten Teil, die Kontinuität zwischen Pharisäern und Rabbinen.

Gegen die weithin vertretene Ansicht lassen sich eine Reihe von Argumenten anführen:

Nur zwei aus der Mischna bekannte Rabbinen werden in früherer Literatur explizit als Pharisäer bezeichnet: Apg 5,34 stellt Rabban Gamaliel (den Älteren) als pharisäisches Mitglied des Rates zu Jerusalem dar; seinen Sohn Simeon ben Gamaliel schildert Josephus als „aus der Stadt Jerusalem, aus einer sehr hervorragenden Familie stammend und zur Schule der Pharisäer gehörig" (Vita 190). Diese zwei Personen sind die einzigen wirklich belegten persönlichen Bindeglieder zwischen den beiden Bewegungen - man müßte natürlich noch fragen, mit welchem Recht die Mischna den älteren Gamaliel als Rabbi adoptiert. Wurde er nur deshalb in die Vorgeschichte des Rabbinats eingefügt, weil er der Ahnherr der Patriarchenfamilie war, oder wäre er wirklich als Rabbi qualifiziert gewesen, wenn es den Titel zu seiner Zeit schon gegeben hätte? Und waren es nur seine persönlichen Einstellungen und Lehren bzw. seine familiären Verbindungen, die ihn qualifizierten, oder hätte man jeden anderen pharisäischen Lehrer der Tempelzeit gleicherweise als Teil der rabbinischen Bewegung betrachtet? Wenn man das frühe Rabbinat als direkte Fortführung des Pharisäismus betrachten kann, ist es andererseits eigenartig, daß Jochanan ben Zakkai, der als der Begründer und erste Führer der rabbinischen Bewegung gilt, nicht mit dem Pharisäismus vor 70 in Verbindung gebracht

14 Siehe z. B. die bekannte Studie von J. Louis Martyn, History and Theology in the Fourth Gospel (New York 1968) $72 \mathrm{ff}$.

15 Der Begriff, im 19. Jahrhundert durch Heinrich Graetz aufgebracht, wird zwar in der Fachliteratur immer seltener verwendet oder in Anführungszeichen gesetzt, doch die damit verbundenen Vorstellungen erweisen sich als sehr zählebig.

16 Clemens Thoma, Der Pharisäismus, in: Johann Maier, Josef Schreiner (Hrsg.), Literatur und Religion des Frühjudentums (Würzburg 1973) 254-272, 270. 
wird $^{17}$. Die Traditionskette in mAbot, die einen Stammbaum der rabbinischen Bewegung zu erstellen sucht, scheint zu den spätesten Schichten dieses Traktates und nicht in die Zeit der Mischna zu gehören ${ }^{18}$; doch nicht einmal dieser Text verbindet wirklich Pharisäismus und Rabbinat. Auch wenn man es immer wieder lesen kann ${ }^{19}$, ist Abot keine pharisäische Schrift.

Namen sind natürlich nicht alles. Zu wenige Juden Palästinas der Jahrzehnte vor 70 sind namentlich bekannt; viele von diesen mögen im Aufstand gegen Rom ihr Leben gelassen haben. Somit ist jedes auf Namen allein gestützte Argument zwar interessant, aber nicht wirklich entscheidend. Wichtiger ist dagegen wohl die Tatsache, daß die Gelehrten der Mischna es fast durchwegs vermeiden, Pharisäer zu erwähnen. Nur ein größerer Abschnitt, mJad 4,6-8, bietet eine Reihe von Kontroversen (zu Fragen von Reinheit und Zivilrecht) zwischen Pharisäern und Sadduzäern. Da die Pharisäer immer das letzte Wort behalten und ihre Meinung mit der der Redaktoren der Mischna übereinstimmt, ist eine gewisse Nähe zwischen Pharisäern und Rabbinen anzunehmen ( $\mathrm{t} J a d$ 2, $19 \mathrm{f}$ bringt ähnliche Kontroversen zwischen Pharisäern und Boethusiern, wohl ein Zweig der Sadduzäer oder auch nur ein anderer Name für sie). Auffällig ist, wie Jochanan ben Zakkai die Sadduzäer fragt (mJad 4,6): „Haben wir nur das gegen die Pharisäer?“ Zumindest rhetorisch stellt er sich damit auf die Seite der Sadduzäer! Man könnte auf Texte von Mischna und Tosefta verweisen, die Sadduzäer oder Boethusier den Ältesten oder Weisen gegenüberstellen (z.B. mPara 3,7f; mMak 1,6; tJoma 1,8). Hier scheinen die Rabbinen den Platz einzunehmen, der in $\mathrm{mJad} 4$ and $\mathrm{tJad} 2$ den Pharisäern vorbehalten ist. Könnte es sein, daß die früheren Pharisäer einfach diesen Namen nicht mehr verwenden wollten, der ihnen vielleicht von Anfang an von außen beigelegt wurde und der für den angestrebten Neuanfang nicht hilfreich war? Ein gewisser historischer Kern dieser These ist durchaus möglich, doch reicht sie gewiß nicht als Gesamterklärung des Phänomens aus. Die rabbinischen Texte, die die Sadduzäer kritisieren und lächerlich machen, sind aus historischer Sicht kaum von Wert; „Pharisäer“ und „Älteste, Weise, Rabbinen“ sind nicht ohne weiteres austauschbare Begriffe. Und sogar wenn wir annehmen dürften, daß viele oder sogar die meisten Rabbinen frühere Pharisäer waren, wollten sie offensichtlich nicht daran erinnert werden. Sie wollten etwas Neues beginnen.

Ein anderer Zugang zur Frage von Kontinuität und Diskontinuität ist eine Analyse der Grundzüge von Lehre und Halakha, die den Pharisäern bzw. den frühen Rabbinen wichtig waren. Doch wissen wir leider nur wenig über die Pharisäer vor 70 . Ihre Betonung von Tradition sowie von Speise- und Reinheits-

17 Jacob Neusner, The Formation of Rabbinic Judaism: Yavneh (Jamnia) from A.D. 70 to 100, in: ANRW II 19,2 (Berlin 1979) 3-42, 30-32.

18 Günter Stemberger, Die innerrabbinische Überlieferung von Mischna Abot, in: Geschichte - Tradition - Reflexion. Festschrift für Martin Hengel, Bd. 1: Judentum, hrsg. von Peter Schäfer (Tübingen 1996) 511-527.

${ }^{19}$ So z.B. R. Travers Herford, The Ethics of the Talmud: Sayings of the Fathers (New York $1962={ }^{3} 1945$ ) 14: "Aboth as a document of Pharisaism". 
gesetzen, sowohl bei Josephus wie auch im Neuen Testament belegt, wird auch von den Meistern der Mischna fortgeführt. Doch die meisten dieser Anliegen sind nicht spezifisch genug, um die direkte Fortsetzung des Pharisäismus im Rabbinat zu beweisen. Diese Anliegen waren mehr oder weniger allen Juden gemeinsam, denen ihre religiöse Tradition wichtig war. Wie heute allgemein vertreten wird, können die in der Mischna und in anderen rabbinischen Texten den Meistern vor 70 zugeschriebenen Positionen nicht ohne weiteres für eine Rekonstruktion der pharisäischen Halakha verwendet werden. Auf diese Weise die Kontinuität nachweisen zu wollen wäre eine petitio principii. Texte aus Qumran bieten Nachrichten über halakhische Auffassungen, die von den Leuten von Qumran abgelehnt werden. Manche dieser Auffassungen, die mit einiger Sicherheit den Pharisäern zugeschrieben werden können, haben enge Parallelen in der rabbinischen Halakha. Doch muß man bei einer Auswertung dieser Parallelen sehr vorsichtig sein; vielfach belegen sie gemeinsame Entwicklungslinien der Halakha, doch nicht schon notwendig identische halakhische Entscheidungen 20.

Soweit wir pharisäische Positionen rekonstruieren können (die sich gewiß auch im Lauf der Geschichte dieser Gruppe in bestimmter Weise verändert haben), scheinen manche Meister der Mischna pharisäische Interessen fortzufühen. Doch ist zu beachten, daß die Hauptakzente der Mischna priesterlich und nicht spezifisch pharisäisch sind. Tempel, Kult und kultbezogene Reinheitsfragen stehen im Mittelpunkt der Mischna und nehmen in ihr den größten Raum ein. Jacob Neusner hat die Leute hinter der Mischna treffend beschrieben als „a cultic sect, a holiness order, expressing the aspirations of lay people to live as if they belonged to the caste of priests, and of priests to live as if the whole country were the Temple“21.

Wenn man alle Belege zusammenfaßt (insgesamt sehr wenig sicheres Material), könnte man, wieder mit Neusner ${ }^{22}$ und anderen Autoren, die in Jabne versammelten Gelehrten nicht als pharisäische Gruppe betrachten, sondern eher als eine große Koalition aller Kräfte, die bereit waren, im Anschluß an die Katastrophe gemeinsame Sache zu machen. „Yavneh was a grand coalition of different groups and parties, held together by the belief that sectarian self-identification was a thing of the past. "23 Diese Definition von Shaye Cohen ist sicher zu optimistisch, wenn

20 Siehe Yaakov Sussmann, The History of the Halakha and the Dead Sea Scrolls, in: Elisha Qimron, John Strugnell, Qumran Cave 4 V: Miqsat Ma'ase ha-Torah (DJD X, Oxford 1994) 179-200 (hebräische Fassung mit ausführlicheren Anmerkungen, in: Tarbiz 59 [1989/90] 11-76); Yaakov Elman, Some Remarks on 4QMMT and the Rabbinic Tradition, Or, When Is a Parallel Not a Parallel?, in: Jobn Kampen, Mosbe J. Bernstein, (Hrsg.), Reading 4QMMT. New Perspectives on Qumran Law and History (SBL Symposium Series 2, Atlanta 1996) 99-128. Siehe auch die anderen Beiträge in diesem Band und die Bibliographie 145-156.

21 Jacob Neusner, Judaism. The Evidence of the Mishnah (Chicago 1981) 119.

22 Jacob Neusner, Early Rabbinic Judaism (Leiden 1975) 64; idem, The Formation of Rabbinic Judaism 37, zu den Schülern des Jochanan ben Zakkai.

${ }^{23}$ Shaye J.D. Coben, The Significance of Yavneh: Pharisees, Rabbis, and the End of Jewish Sectarianism, in: HUCA 55 (1984) 27-53, 50. 
sie historische Fakten beschreiben möchte; doch trifft sie gut die idealisierte Auffassung, die die Redaktoren der Mischna von den Absichten der Gründer von Jabne hatten.

\section{Lehrhaus, Gericht, Sanhedrin}

Wenden wir uns nun der Frage zu, ob die Gruppe der frühen Rabbinen in Jabne mehr als eine lose Ansammlung von Toragelehrten war, einen institutionellen Charakter hatte und, wenn ja, wie dieser zu definieren ist.

Schürer und seine Bearbeiter zögern, welche genauen Funktionen sie der in Jabne versammelten Gruppe von Toragelehrten zuschreiben sollen, betrachten sie aber als eine Art offizieller Einrichtung. Schon zitiert wurde der Abschnitt, wonach der "Gerichtshof“ (englisch „academy“) von Jamnia „ein Kollegium von Gelehrten“ war, „das schwerlich von der römischen Behörde eine weitgehende Autorisation hatte", tatsächlich aber die Stelle des alten Synhedriums von Jerusalem als oberster Gerichtshof von Israel einnahm und voll in die Rechte Jerusalems nachfolgte, wo nicht Ausnahmen explizit festgestellt wurden ${ }^{24}$.

Demnach wäre die Versammlung der Gelehrten zu Jabne als Nachfolgeorganisation des Sanhedrin zu Jerusalem zu verstehen, wenn auch nicht voll von den Römern dazu autorisiert. Bedeutet das, daß die römische Verwaltung ihnen sehr wohl einen Auftrag erteilte, die Rabbinen jedoch über diesen hinausgingen? Oder haben die Römer nur stillschweigend geduldet, was sich in Jabne entwickelte? Worauf stützten sich die Rabbinen, falls sie tatsächlich den Anspruch erhoben, den Jerusalemer Sanhedrin fortzuführen? Eine Beauftragung durch „das jüdische Volk" ist kaum denkbar. Sollte man sich die Rabbinen oder zumindest ihren Kern als Überlebende des früheren Sanhedrin vorstellen, die auch ohne direkte Autorisation ihre alten Funktionen weiterführen und ein Vakuum füllen wollten, oder als selbsternannte „Exilsregierung“, die nur darauf hinarbeiten konnte, im Lauf der Zeit sowohl im Volk wie auch bei der römischen Besatzung Anerkennung zu finden? Bevor wir nach Belegen für einen solchen Führungsanspruch der Rabbinen fragen, ist kurz auf das Problem des Sanhedrin vor 70 einzugehen, dessen Nachfolge die Rabbinen zu Jabne angetreten haben sollen.

Frühere Diskussionen des Sanhedrin vor 70 bemühen sich vor allem, die verschiedenen Quellen (Josephus, Neues Testament, rabbinische Texte) miteinander auszugleichen und zu entscheiden, ob es nur einen Sanhedrin oder mehrere Institutionen gab, die diesen Namen trugen, aber verschieden zusammengesetzt waren und verschiedene Kompetenzen hatten ${ }^{25}$. Die englische Neufassung Schürers formuliert: „if by 'the Great Sanhedrin' is meant a body officially recognized by the occupying power and endowed with competence in juridical and administrative

24 Schürer, G I $657 \mathrm{f}$. = H I $525 \mathrm{f}$.

25 Schürer, G II $237 \mathrm{ff}$. ist in H II 199ff. in einigen Passagen stärker revidiert worden. 
matters and in legal exegesis, this was a single institution under the presidency of the High Priest (in the absence of a king)" 26 ; die Annahme rabbinischer Texte, daß die Führer der proto-rabbinischen Schulen jeweils Vorsitzender und Stellvertreter der Institution waren, wäre demnach eine anachronistische Verzeichnung. In der Fortsetzung stimmen beide Fassungen überein: „Nach der Zerstörung Jerusalems im Jahre 70 ist ohne Zweifel das Synedrium in seiner bisherigen Form aufgehoben... die Regierungsgewalt wurde jetzt direkter von den Römern in die Hand genommen." Das neue Zentrum in Jabne war „etwas wesentlich anderes als das alte Synedrium... ein juristisches Tribunal, dessen Entscheidungen zunächst nur theoretische Bedeutung hatten. Und obwohl auch dieses bald wieder zu einer großen Macht über das jüdische Volk dadurch gelangte, daß es eine wirkliche, teils zugestandene, teils usurpierte Gerichtsbarkeit über dasselbe ausübte (englisch: ,partly with Roman consent and partly without it ${ }^{6}$ ), so hat doch das rabbinische Judentum stets ein deutliches Bewußtsein davon gehabt, daß das alte ,Synedrium" aufgehört hat zu existieren." 27

Neuere Forschung hat in Frage gestellt, ob ein Sanhedrin als permanente Einrichtung vor 70 überhaupt je existiert hat. David Goodblatt stellt nach gründlicher Analyse der Quellen und der Forschungsgeschichte fest: „Only Acts appeared to offer a clear picture of an ongoing Judean council known as the sunedrion. But the value of its testimony is questioned by many scholars, the more so in the absence of corroboration more familiar with Judean realities. "28 Natürlich gab es Leute, die in der Gesellschaft Judäas die Macht ausübten. „But what is missing is clear evidence for a regular and institutionalized coming together of these people, i.e. a council. A conciliar institution cannot be excluded, given the limitations of the available evidence. But I think conciliar supremacy can be excluded. " 29

Mehrere andere Autoren sind schon früher zu ähnlichen Schlüssen gekommen, wenn auch weniger detailliert begründet. Hier sind vor allem Joshua Efron, Martin Goodman und Ed P. Sanders zu nennen ${ }^{30}$. Gegen die Mehrheitsmeinung, wie er sie nennt, stellt Sanders fest, daß "the theory of party representation, of democratic voting, and of legislation for all Israel passed by the Sanhedrin - a permanent body of legislators and judges, deciding all capital cases and voting on correct observance of the law, even sending emissaries throughout the world to communicate decrees - is completely without basis" 31 . Daß es in Jerusalem einen Stadtrat

26 Schürer, H 208. Sachlich stimmt die Position mit der von G II $258 \mathrm{f}$. überein.

27 Schürer, G II $247 \mathrm{f}$. = E II $208 \mathrm{f}$. Als Beleg für das Ende des Sanhedrin wird mSota 9,11 zitiert.

${ }^{28}$ David Goodblatt, The Monarchic Principle. Studies in Jewish Self-Government in Antiquity (TSAJ 38, Tübingen 1994) 129.

29 Ibid. 130 .

30 Joshua Efron, The Great Sanhedrin in Vision and Reality, in: ders., Studies on the Hasmonean Period (SJLA 39, Leiden 1987) 287-338; Martin Goodman, The Ruling Class of Judaea (Cambridge 1987) $114 \mathrm{ff}$.; Ed P. Sanders, Judaism. Practice \& Belief 63BCE - 66CE (London 1992); siehe auch James S. McLaren, Power and Politics in Palestine (JSNT.S 63, Sheffield 1991).

31 Ed P. Sanders, Judaism 481. 



daß dessen Entscheidungen in gewissen Fällen auch außerhalb von Jerusalem angenommen wurden, was aber nicht mit der Frage des Sanhedrin zu vermengen ist.

Was vor zehn bis zwanzig Jahren noch die Mehrheitsmeinung darstellte, ist somit durch neuere Forschung völlig in Frage gestellt worden. Es hat sich ein neuer Konsens gebildet, auch wenn es natürlich noch immer Autoren gibt, die an der älteren Position festhalten. Wenn er die historische Wirklichkeit trifft, bedeutet das, daß die frühen Rabbinen hinsichtlich des Sanhedrin keine frühere Institution nachahmen und fortführen konnten. Das schließt natürlich nicht von vornherein die Möglichkeit aus, daß die in Jabne versammelten Gelehrten versuchten, eine neue Form nationaler Führung zu etablieren, indem sie verschiedene ihnen aus der Zeit vor 70 oder auch aus der Bibel bekannte Vorbilder (etwa die von Mose eingesetzten siebzig Ältesten, Num 11,16) miteinander kombinierten.

Von größtem Interesse ist eine R. Simeon ben Azzai zugeschriebene Aussage (mJad 3,5; 4,2; mZeb 1,3), die also in die Zeit Gamaliels II. gehört: „Mir ist überliefert aus dem Mund der 72 Ältesten an dem Tag, an dem sie Rabbi Eleazar ben Azaria einsetzten “. Die versammelten Rabbinen besprechen Reinheitsgesetze und nichts, was mit Fragen nationaler Führung zu tun hat. Doch fältt die symbolische Zahl der Ältesten auf, die damals versammelt gewesen sein sollen, und die als Vertretung von ganz Israel verstanden werden könnte, seien es eben die von Mose eingesetzten Ältesten (ergänzt um Gamaliel und Eleazar) oder je sechs Vertreter der zwölf Stämme Israels (wie die 72 Übersetzer der Tora im Aristeasbrief). Will man hinter dieser Zahlenangabe eine historische Basis sehen, würde sie wohl anzeigen, daß die Rabbinen in Jabne sich der nationalen Bedeutung dessen, was sie taten, sehr bewußt waren. Ob es damals in der jungen Bewegung schon so viele hervorragende Mitglieder gab (noch in späterer Zeit kann man die Zahl der Rabbinen in Palästina je Generation vielleicht mit 150-200 ansetzen ${ }^{32}$ ), ist allerdings höchst fraglich; man könnte in der Zahl auch eine spätere Stilisierung der Anfänge zu Jabne sehen. Sie wäre mit dem idealen größten Gerichtshof von 71 Mitgliedern $\mathrm{zu}$ verbinden, der laut $\mathrm{mSanh}$ 1,5 die höchsten nationalen Entscheidungen trifft (das Gericht über einen ganzen Stamm oder eine abgefallene Stadt, über einen Lügenpropheten oder den Hohenpriester, Kriegsbeschluß usw.). Laut tKel BB 2,4 sind es sogar 85 Älteste, die mit Rabban Gamaliel eine Reinheitsfrage entscheiden (vielleicht in Anlehnung an die 85 Mitglieder der "Großen Synagoge"?); in Sifre Num $\ 124$ behandeln in Jabne 38 Älteste wiederum eine Reinheitsfrage.

$\mathrm{Daß}$ gerade dort, wo so viele Rabbinen gemeinsam Entscheidungen fällen, nie Fragen der nationalen Führung oder auch nur gravierende Gerichtsfälle anstehen, zeigt, daß die Redaktoren dieser Texte kaum an die Fortführung eines Jerusalemer Sanhedrin dachten ${ }^{33}$. Texte wie mMak 1,10 und mSota 9,11 sagen sogar explizit,

32 Für verschiedene Schätzungen siehe L. I. Levine, The Rabbinic Class of Roman Palestine in Late Antiquity (Jerusalem 1989) 66-69.

33 David Goodblatt, The Monarchic Principle 232 ff., bespricht jene Texte, die die Versammlung einer größeren Anzahl von Rabbinen erwähnen. 
$\mathrm{daß}$ es seit Zerstörung des Tempels keinen Sanhedrin mehr gibt (zumindest was die Kapitalgerichtsbarkeit angeht). Ein Text scheint dagegen einen rabbinischen Sanhedrin anzunehmen, wenn nämlich Eleazar ben R.Zadoq in der Diskussion um die Sitzordnung im Sanhedrin sagt: „Als Rabban Gamaliel in Jabne saß, saßen mein Vater und ein anderer zu seiner Rechten und die Ältesten saßen zu seiner Linken“ (tSanh 8,1). Doch fügt der Text redaktionell verschiedene Elemente zusammen; er mag das Zeremoniell größerer rabbinischer Versammlungen in Analogie zum Sanhedrin sehen, belegt aber nicht, daß man zu Zeiten Gamaliels diese Versammlungen als Sitzung eines Sanhedrin unter Leitung des Patriarchen verstand $^{34}$. Mit David Goodblatt läßt sich zusammenfassen: „no tannaitic source alludes to a contemporary sanhedrin" 35 .

Wenn die Rabbinen zu Jabne sich nicht als Sanhedrin verstanden, ist vielleicht ein anderes Selbstverständnis nachzuweisen, das einen Führungsanspruch über Israel vermittelt? Zwar sprechen tannaitische Texte von einem beit din zu Jabne bzw. von Gruppenentscheidungen im „Weinberg von Jabne“, doch nirgends erkennt man einen klaren Anspruch nationaler Führung. Das gilt auch von den Taqqanot von Jabne. Mischna und Tosefta schreiben auch schon den Vorläufern der Rabbinen aus der Zeit des Tempels Taqqanot zu (Hillel, Gamaliel der Ältere usw.), ohne daß klar ist, aufgrund welcher Autorität und für wen diese Verfügungen, so sie überhaupt historisch sind, erlassen wurden. In Jabne werden Taqqanot sowohl Jochanan ben Zakkai als Einzelperson wie auch einer anonymen Mehrzahl von Meistern („,sie setzten fest“) zugeschrieben ${ }^{36}$. Die von diesen Taqqanot betroffenen Bereiche sind nicht gerade wichtige Elemente des täglichen Lebens, in denen Führungskompetenz für Israel als ganzes gefragt war (z. B. mRH 4,1-4: Schofar, Lulab, Zeugnis in Bezug auf den Neumond usw.). Auch hier ist zu fragen, für wen diese Entscheidungen gedacht waren, ob sie zuerst einmal nur das innere Verhalten der rabbinischen Gruppe bestimmten und vielleicht erst später immer mehr auch das allgemeine jüdische Leben prägten. Tatsächliche Auswirkungen dieser Taqqanot sind nicht zu ermessen und damit ist auch die Frage eines eventuell damit verbundenen Führungsanspruches kaum zu beurteilen. Wenn Taqqanot wechselweise einzelnen führenden Rabbinen und dann wieder einem anonymen Plural, d.h. idealerweise der Gesamtheit der Rabbinen zugeschrieben werden, fragt sich, ob der einzelne aus eigener Autorität oder nur im Namen der Gruppe entscheidet oder ob die Rabbinen als Beratungsgremium des Führers ihrer Bewegung fungieren. Das würde dem Modell entsprechen, wonach heute gern der Sanhedrin der Zeit vor 70 verstanden wird. Damit stellt sich die Frage nach den Anfängen des Patriarchats.

${ }^{34}$ Siehe Martin Jacobs, Die Institution des jüdischen Patriarchen (TSAJ 52, Tübingen 1995) 62-64. Vgl. David Goodblatt, The Monarchic Principle $189 \mathrm{f}$.

35 David Goodblatt, The Monarchic Principle 237.

36 Siehe Avrabam Aderet, From Destruction to Restoration. The Mode of Yavneh in ReEstablishment of the Jewish People (hebräisch, Jerusalem 1990). 


\section{Die Anfänge des Patriarchats}

Traditionelle Geschichtsschreibung geht davon aus, daß sofort oder zumindest bald nach 70 der Führer der rabbinischen Bewegung als Patriarch der Führer auch des jüdischen Volkes in Palästina (oder auch der Diaspora im römischen Reich) wurde, von der römischen Behörde ebenso anerkannt wie von der jüdischen $\mathrm{Ge}$ meinschaft. Seine reale, vielleicht auch schon offizielle Machtposition hätte das durch den Untergang des Hohenpriestertums entstandene Machtvakuum ausgefüllt. $\mathrm{Daß}$ es dafür andere Kandidaten mit traditionellerem Anspruch gegeben hätte, sei es ein Vertreter einer hohenpriesterlichen Familie oder noch mehr Agrippa II. ${ }^{37}$, wird dabei kaum einmal beachtet. Innerjüdische Anerkennung wäre besonders dann denkbar, wenn der Führer der rabbinischen Schule direkt an eine einflußreiche pharisäische Bewegung hätte anknüpfen können, wofür aber, wie schon gesagt, Belege fehlen. Wie die Anerkennung einer neuen Führung ohne guten traditionellen Rückhalt durch das Volk hätte gewonnen und ausgedrückt werden sollen, läßt sich schwer sagen; das hätte auf jeden Fall einen längeren Zeitraum beansprucht. Direktbeauftragung durch die römische Verwaltung wäre natürlich rasch und problemlos durchführbar; doch bleibt die Frage, wie die Römer gerade auf Jochanan ben Zakkai bzw. Gamaliel gekommen wären. Die rabbinische Tradition hat hier eine einfache, wenn auch historisch fragwürdige Lösung - der Eindruck, den Jochanan ben Zakkai persönlich auf Vespasian gemacht hat ${ }^{38}$.

Konkrete Aussagen, daß schon Jochanan oder Gamaliel größere Autorität als die des Führers der ersten Rabbinen hatten, sind in der frühen rabbinischen Literatur kaum zu finden. Im wesentlichen sind es die schon genannten Texte mEd 7,7 (Rabban Gamaliel reist nach Syrien, um eine Erlaubnis/Autorisierung zu erlangen) und mJad 3,5, die Einsetzung von Eleazar ben Azaria - daß הושיבו setzung als Patriarch anstelle von Rabban Gamaliel bedeutet, ist allerdings erst das talmudische Verständnis des Textes, der wohl einfach von der Aufnahme Eleazars in das rabbinische Gericht bzw. als stimmfähiges Mitglied in die Schule spricht ${ }^{39}$.

Angesichts der spärlichen Quellen für die frühe Zeit ist es verständlich, daß auch in der jüngsten Forschung die Meinungen auseinandergehen, wer der erste von den Römern anerkannte jüdische Patriarch war: War dies schon Gamaliel, wie z. B. David Goodblatt vertritt, oder erst Jehuda ha-Nasi etwa cin Jahrhundert später, wie Martin Jacobs argumentiert ${ }^{40}$ ? Für beide Lösungen gibt es keine wirklich

37 Dazu siehe Seth Schwartz, Josephus and Judaean Politics (CSCT 18, Leiden 1990) 110169.

${ }^{38}$ Für eine gründliche Quellenanalyse siehe Peter Schäfer, Die Flucht Johanan b. Zakkais aus Jerusalem und die Gründung des „Lehrhauses“ in Jabne, in: ANRW II 19,2 (Berlin 1979) 43-101.

39 Siehe die grundlegende Untersuchung von Robert Goldenberg, The Deposition of Gamaliel II: An Examination of the Sources, in: JJS 23 (1972) 167-190; Nachdruck in: William S. Green (Hrsg.), Persons and Institutions in Early Rabbinic Judaism (Missoula 1977) 9-47.

40 David Goodblatt, The Monarchic Principle 176ff.; ders., The Origins of the Roman Recognition of the Palestinian Patriarchate (hebr.), in: Studies in the History of the Jewish People 
stichhaltigen Beweise. Da aber unter Jehuda ha-Nasi enge Kontakte zu den römischen Behörden schon selbstverständlich sind und er auch vom jüdischen Volk offenbar ohne ernsthafte Opposition als Führer anerkannt wird, ist wohl eine gewisse Vorgeschichte der Institution anzunehmen; sie reicht wahrscheinlich in die Zeit nach dem Bar Kokhba-Aufstand zurück, als endgültig klar war, daß eine Rückkehr zur alten Ordnung nicht so schnell kommen werde. Eine Lösung irgendwo zwischen den Positionen von Goodblatt und Jacobs scheint mir daher plausibel. Dabei bleibt noch immer offen, was zuerst kam - die innerjüdische oder die römische Anerkennung. Womit die Funktion ihren Anfang nahm, ist ebenfalls ungewiß, wenn man von der Leitung der rabbinischen Schule oder des wichtigsten rabbinischen Zentrums als wohl selbstverständlich absieht. Auf Einzelheiten einzugehen ist hier nicht der Platz, doch sei zumindest ein Element genannt, wo man wohl schon sehr früh mit dem Einfluß des Patriarchen im Judentum rechnen muß - die Kalenderautorität. Das war von Anfang an ein praktisches Bedürfnis aller jüdischen Gemeinden, die die traditionellen Feste im geregelten Ablauf begehen wollten. Nach dem Wegfall der Tempelbehörden, deren Aufgabe dies zuvor war, mußte man dankbar sein, wenn sich eine kompetente Instanz fand, die diese Aufgabe zu übernehmen imstande und bereit war. Wie wichtig diese Frage war, zeigen die zahlreichen rabbinischen Aussagen zum Thema. Die einzige Alternative wäre die Annahme eines festen Kalenders schon um 70 - die Beobachtung des Neumonds und die Funktion eines Kalendergerichts wäre dann rein rituell zu verstehen und nicht als praktische Notwendigkeit -, doch dafür gibt es keine Belege.

\section{Der Einfluß der Rabbinen in der Zeit von Jabne}

Der bisherige Überblick hat verdeutlicht, daß wir für die Jahrzehnte nach 70 keine wirklichen Belege haben, daß die rabbinische Bewegung zu Jabne sich in direkter Kontinuität zu den Pharisäern vor 70 verstand, daß sie den Sanhedrin oder sonst eine zentrale nationale Führung fortsetzen wollte oder daß es das Amt des Patriarchen gegeben habe. Könnte man aber nicht zumindest annehmen, daß auch ohne formale Führungsrolle die Rabbinen schnell zu den neuen geistigen Autoritäten wurden, die unter den ernsthaft religiös Gesinnten bald ihren Anhang hatten? Dann könnte man folgenden Zitaten aus Schürer doch in den großen Zügen zustimmen, auch wenn man keine so direkte Rückbindung der Rabbinen an die Pharisäer annimmt:

„Im letzten Jahrhundert vor der Zerstörung Jerusalems hatten die pharisäischen Schriftgelehrten schon so unbedingt die geistige Herrschaft, daß das große Syne-

and the Land of Israel 4, hrsg.von Uriel Rappaport (Haifa 1978) 89-102; Martin Jacobs, Die Institution 349. Siehe auch Lee I. Levine, The Status of the Patriarch in the Third and Fourth Centuries: Sources and Methodology, in: JJS 47 (1996) 1-32, $28 \mathrm{f}$, der die Linie von einer frühen Stufe der Anerkennung Gamaliels bis zur vollen Blüte unter Jehuda ha-Nasi zieht. 
drium trotz seiner gemischten Zusammensetzung aus Pharisäern und Sadduzäern in der Praxis doch an das von den Pharisäern entwickelte Recht sich anschloß... Die Schriftgelehrten sind - wenn auch nicht auf Grund formeller Anerkennung, so doch tatsächlich - die Gesetzgeber. Ganz besonders gilt dies von der Zeit nach der Zerstörung des Tempels... Der allein maßgebende Faktor waren nun die rabbinischen Gesetzeslehrer mit ihrer rein geistigen Autorität. Sie, die schon früher tatsächlich das Recht festgestellt hatten, wurden jetzt immer mehr auch formell als die entscheidenden Autoritäten anerkannt. Ihr Ausspruch genügt, um festzustellen, was gültiges Gesetz ist. “41 "Die gesetzgebende Gewalt der Rabbinen ist für das Zeitalter der Mischna eine so selbstverständliche Sache, daß sie auch schon für die Zeit vor der Zerstörung des Tempels ohne weiteres vorausgesetzt wird." 42

"Nach dem Untergang des jüdischen Staatswesens im Jahre 70 hat auch in dieser Beziehung [d.h. im Gerichtswesen] die Autorität der Rabbinen an selbständiger Bedeutung noch gewonnen. Wie man sie jetzt als selbständige Gesetzgeber anerkannte, so hat man sie auch als selbständige Richter anerkannt. Man fügte sich freiwillig ihrem Urteil, mochten sie nun als Kollegium oder als Einzelrichter entscheiden." 43

Die Behauptung unmittelbarer Anerkennung der geistigen Autorität der Rabbinen durch das Volk steht gegen die These, daß die Rabbinen eine private Bewegung ohne viel Rückhalt unter den "normalen Juden“ waren und sich erst über die Jahrhunderte als die geistige Führung des Judentums etablieren konnte. Mischna und Tosefta sind weitgehend theoretisches Recht, auch von jenen nicht verwirklichbar, die bereit gewesen wären, sich daran zu halten. Auch dort, wo die Texte tatsächliche konkrete Entscheidungen (מעט (מט) wiedergeben wollen, betrifft dies nur relativ unbedeutende Einzelheiten, in denen sich bestimmte Personen aus dem Umfeld der Rabbinen freiwillig an diese wenden. Wieweit die rabbinischen Diskussionen das praktische Leben bestimmt haben, läßt sich so schwer sagen. Was die Verwaltung von Gemeinde, Ortsgericht und Wohlfahrtspflege betrifft, scheinen sich die Rabbinen erst ab dem 3. Jahrhundert vermehrt um Einfluß bemüht zu haben; dasselbe gilt von Schule und Synagoge. Welche Vorläufer diese Entwicklung in früherer Zeit gehabt hat, ist schwer festzustellen.

In seiner Analyse der Entwicklung der Gesetze der Mischna kommt Jacob Neusner zum Ergebnis, daß der Zeit zwischen 70 und 132 sehr wenig praktisch anwendbares Material (vor allem in den Ordnungen Naschim und Neziqin - Personen-, Zivil- und Strafrecht) zuzuordnen ist ${ }^{44}$; und sogar das Wenige hat sich wohl kaum außerhalb der rabbinischen Gruppe ausgewirkt. Zweifel am konkreten Einfluß der Rabbinen auf das tägliche jüdische Leben ergeben sich jedoch nicht nur aus dem Studium der rabbinischen Quellen. Auch die erhaltenen Dokumente aus dieser Zeit - Ehe- und Scheidungsverträge und vermögensrechtliche

41 Schürer, G II $382 \mathrm{f} .=$ H II 331.

42 Schürer, G II $383=$ H II 332.

43 Schürer, G II $387=$ H II 335.

${ }^{44}$ Jacob Neusner, Judaism. The Evidence of the Mishnah 111-119. 
Urkunden - zeigen trotz aller Nähe zu gewissen auch in den rabbinischen Texten bezeugten Normen, daß man sich (zumindest in den erhaltenen Belegen) nicht an die rabbinischen Vorstellungen gehalten hat ${ }^{45}$.

Es ist durchaus denkbar, daß frühe Rabbinen als Einzelpersonen einen gewissen Rückhalt im Volk gehabt haben, als geistige Führer, Charismatiker, Heilige; doch die Bewegung als solche konnte kaum mit Einfluß auf das Volk rechnen. Das ergibt sich nicht nur aus dem bisher Gesagten. Das Hauptargument gegen die sofortige Annahme der rabbinischen Führerschaft durch das Volk ist der Bar KokhbaAufstand, der wohl kaum rabbinischen Bestrebungen entsprach, auch wenn ein Rabbi Aqiba Bar Kokhba als messianische Gestalt verstanden haben soll. Die Jahrzehnte zwischen 70 und 132 sind eine Zeit des Übergangs. Es ist kein Zufall, wenn Schürer seine Geschichte bis zum Jahre 135 führt, wie dies auch verschiedene neuere Darstellungen machen, andererseits z.B. Michael Avi-Yonah seine Geschichtsdarstellung im Jahr 135 einsetzen läßt $\mathrm{t}^{46}$. In vielerlei Hinsicht gehören die Jahrzehnte bis 135 noch zur Zeit des Zweiten Tempels und zur alten Ordnung. Was immer es schon an neuen Entwicklungen gegeben hat, war nicht mehr als eine Saat, die erst viel später Früchte brachte.

45 Darauf hat auf dieser Tagung nachdrücklich Hannah Cotton hingewiesen. Eine Übersicht des derzeit bekannten Materials: Hannab M. Cotton, W. E. H. Cockle, Fergus G. B. Millar, The Papyrology of the Roman Near East: A Survey, in: JRS 85 (1995) 214-235. Zur Diskrepanz der erhaltenen Eheverträge zum rabbinischen Recht siehe auch Michael Satlow, Reconsidering the Rabbinic ketubah Payment, in: Shaye J. D. Coben (Hrsg.) The Jewish Family in Antiquity (BJS 289, Atlanta 1993) 133-151: „None of the extant Jewish marriage contracts from the rabbinic period show any familiarity with the rabbinic ketubah payment" (141). Ähnlich Hayim Lapin, Early Rabbinic Civil Law and the Literature of the Second Temple Period, in: JSQ 2 (1995) 149-183.

46 Michael Avi-Yonah, Geschichte der Juden im Zeitalter des Talmud (SJ 2, Berlin 1962). 
